\title{
ANÁLISIS DE PLANEAMIENTO DE PROTOCOLOS CAN CON CALIDAD DE SERVICIO
}

\author{
Abel Crespo $^{1} \quad$ Walter Grote H. ${ }^{2}$ \\ Recibido el 6 de abril de 2005, aceptado el 28 de junio de 2005
}

\begin{abstract}
RESUMEN
El protocolo de comunicaciones diseñado para redes CAN (Controller Area Network) procesa mensajes sensibles e insensibles al tiempo real. Para los primeros se aplica planeamiento para que sean transferidos al nodo destino en tiempo válido. La segunda categoría de mensajes admite retardos y hasta algún nivel de pérdidas que, en caso de ser incorporadas al diseño, permiten disminuir las tasas de transmisión, lo que facilita la sincronización de los nodos. Se propone un diseño en que el planeamiento solo se aplica a mensajes de tiempo real y se analiza el comportamiento en valor medio de las tareas que no son de tiempo real.
\end{abstract}

Palabras clave: CAN, Controller Area Network, tiempo real, retardo promedio, planeamiento.

\begin{abstract}
The CAN protocol transfers time sensitive and unrestricted time messages among nodes. To deliver messages within their useful lifetime to destination, priority scheduling is part of the design process. However, some messages in real systems may have relaxed time constraints, allowing even for occasional losses. Allowing for packet losses of these messages, lower data rates can be achieved, thus solving synchronization difficulties at the receiving nodes. In this publication we propose an algorithm that schedules high priority messages that need to fulfill real time constraints, while it guarantees these constraints on lesser priority messages only on the average.
\end{abstract}

Keywords: CAN, Controller Area Network, real time, average delay, scheduling.

\section{INTRODUCCIÓN}

En el caso general los mensajes transferidos por el bus en un sistema CAN pueden clasificarse en dos categorías bien definidas: mensajes con restricciones de tiempo real y mensajes sin restricciones de tiempo real. En la primera categoría es necesario aplicar criterios de planeamiento (scheduling, [1]) a fin de garantizar que cada mensaje sea transferido al nodo destino dentro del tiempo de validez del mismo (vida útil del mensaje). Mensajes que ocupan la segunda categoría son aquellos que admiten retardos y hasta algún nivel de pérdidas, sin que por ello afecte el normal funcionamiento del sistema.

En la actualidad, cuando se diseña un sistema de control distribuido se tiene como premisa el planeamiento de todas las tareas, sin importar el tipo de mensaje de que se trate, [1], [2]. La consecuencia de esta estrategia es que se debe incrementar la tasa de transmisión, lo cual complica la sincronización y correcta lectura de los mensajes por parte de los receptores. Adicionalmente, el aumento de la tasa de transmisión fragiliza todo el sistema en cuanto ocurren errores que incrementan las retransmisiones, lo cual comúnmente no se considera en el diseño [3]. Una alternativa válida sería proveer de criterios de planeamiento sólo a aquellos mensajes cuya vida útil es relevante para el correcto funcionamiento del sistema y analizar el comportamiento en valor medio de las tareas que no son de tiempo real [4]. A continuación se analizará en detalle esta propuesta.

En la próxima sección se introducirán aspectos generales del protocolo CAN que tienen relación con el modo como se implementa la priorización de mensajes en el acceso al canal compartido. Posteriormente se aplicará el criterio de planeamiento a los mensajes con restricciones de tiempo real de un conjunto mayor de mensajes, en que a los restantes no es necesario aplicar este criterio en forma rígida. Se apreciará que si se desea mantener el criterio de planeamiento para los mensajes restantes que no

1 Facultad de Ingeniería - UNLPam, crespoa@ing.unlpam.edu.ar, La Pampa, Argentina.

2 Departamento de Electrónica - UTFSM, wgrote@elo.utfsm.cl, Casilla Postal 110-V, Valparaíso, Chile. 
tienen la restricción imperativa de tiempo de transferencia menor al tiempo de vida útil del mensaje, será necesario aumentar la tasa de transmisión. Luego se calcularán los tiempos promedios de respuesta para todos los mensajes y finalmente en la última sección se presentarán las conclusiones.

\section{PROTOCOLO DE ACCESO DE CAN}

CAN es una arquitectura simple de bus que opera en modo "multimaestro". Los nodos conectados al bus interpretan dos niveles lógicos denominados "dominante" y "recesivo", respectivamente. Esta denominación de los niveles lógicos responde al hecho de que un nivel dominante ( 0 lógico) sobrescribe un nivel recesivo (1 lógico), por lo que el bus, desde el punto de vista lógico, se comporta como una compuerta "y" cableada (wired-and).

Cada mensaje intercambiado entre dos nodos en una red CAN está compuesto de dos campos. Un campo es el identificador de 11 bits de longitud y el otro campo de datos es opcional y puede ser de hasta 8 bytes de longitud, [5]. El campo identificador (ID) sirve a dos propósitos:

1. Identifica el dato que está siendo transmitido en el bus, por lo que existe una relación directa y no ambigua entre dato e identificador.

2. Permite resolver conflictos en caso de múltiple acceso. El mecanismo de acceso al medio empleado por CAN es CSMA/DCR (Carrier Sense MultiAccess with Deterministic Collision Resolution). Los nodos retrasan su transmisión cuando detectan la condición del bus como "ocupado", lo cual ocurrirá mientras otro nodo transmite. Cuando se detecta la condición "bus ocioso", un nodo puede comenzar a transmitir y el conflicto de múltiple acceso es resuelto a través de la comparación entre bits de los identificadores. Mientras no exista diferencia entre el nivel transmitido y el nivel percibido, la resolución del arbitraje no habrá finalizado.

En síntesis, conflictos de múltiple acceso se resuelven según las siguientes reglas:

- Cada estación monitorea el bus de acceso compartido antes de proceder a transmitir un mensaje. En caso de encontrar el bus ocioso, la estación que tiene un mensaje que transmitir procede a hacerlo.

- Durante la transmisión del identificador, la estación monitorea el medio simultáneamente. Si una de las estaciones transmite un bit recesivo y monitorea en el bus un bit dominante, inmediatamente concluye su transmisión y pasa al estado de receptor activo.

- El nodo que posea el menor valor numérico de identificador ganará el acceso al bus. Nótese que es el identificador del mensaje el que determina la prioridad; la procedencia del mensaje -esto es, qué nodo lo emite- no es de relevancia en el contexto de CAN.

Este mecanismo de arbitraje tiene las siguientes ventajas:

- Es inherentemente no destructivo, debido a que el nodo que gana el acceso al bus continúa su transmisión sin retardo.

- En caso de múltiple acceso permite priorizar un mensaje urgente. Un mensaje con un ID de valor numérico bajo tendrá alta prioridad.

Finalmente, un mensaje en servicio no puede ser desalojado del bus por un mensaje de mayor prioridad.

\section{ANÁLISIS DE PLANEAMIENTO DE CAN}

Como se señalaba en la sección introductoria, los análisis que se realizan de los protocolos que permiten controlar variables de procesos en tiempo real consideran que los mensajes que se transmiten entre nodos de esa red tienen un tiempo de vida útil y se generan con una cierta frecuencia. El estudio más aceptado es aquél en que se asegura que todos los mensajes de la red pueden ser transmitidos antes de que queden obsoletos. Sin embargo, últimamente se ha cuestionado la validez de este argumento, debido a que resulta en un diseño sobredimensionado, en que variables de poca importancia, que perfectamente pueden experimentar una mayor demora o incluso una pérdida, determinan la mínima tasa de transmisión de la red [3]. Por otro lado, por lo general se procura reducir la tasa de transmisión para facilitar la sincronización de los nodos y asegurar una reducción de errores de canal por efectos de ruido. En este trabajo se propone negociar la necesidad de asegurar de que todas las variables transmitidas lleguen 
a destino en el tiempo de vida útil, manteniéndose esta condición sólo para aquellas para las cuales resulta imprescindible. Además, se calcula el efecto que tiene el hecho de que las variables menos relevantes se transmitan sin cumplir rigurosamente con la exigencia.

Para los efectos de estudio, se considerará un sistema CAN conformado por un número determinado de nodos, cada uno de ellos con capacidad de generar y recibir mensajes para controlar parte del proceso de control distribuido. Existe un total de 9 mensajes distintos, $7 \mathrm{de}$ ellos con características de tiempo real y 2 tipos de mensajes que toleran algún grado de pérdidas y retardos tal como se explicó en la primera sección. La Tabla 1 muestra todos los mensajes ordenados por la prioridad asignada.

La segunda columna de la tabla muestra el número de bytes de datos para cada tipo de mensaje definido. La tercera columna especifica el período de arribo de los mensajes al buffer de transmisión. En la última columna se especifica el tiempo de vida útil de cada mensaje, $\mathrm{Di}^{3}$. Sin embargo, para los mensajes 8 y 9 (sombreados) los valores de Di y Ti constituyen valores promedio; en otras palabras, se permite que en ocasiones los mensajes no lleguen a destino en el tiempo planeado indicado en la Tabla 1.

Tabla 1 Requerimientos para la transmisión de mensajes.

\begin{tabular}{|c|c|c|c|}
\hline $\operatorname{Pr}$ & Bytes & $\operatorname{Ti}(\mathrm{s})$ & $\operatorname{Di}(\mathrm{s})$ \\
\hline 1 & 4 & 0.05 & 0.005 \\
\hline 2 & 1 & 0.005 & 0.005 \\
\hline 3 & 1 & 0.005 & 0.005 \\
\hline 4 & 2 & 0.005 & 0.005 \\
\hline 5 & 1 & 0.005 & 0.005 \\
\hline 6 & 1 & 0.005 & 0.005 \\
\hline 7 & 1 & 0.005 & 0.005 \\
\hline 8 & 3 & $0.005^{*}$ & $0.005^{*}$ \\
\hline 9 & 3 & $0.005^{*}$ & $0.005^{*}$ \\
\hline
\end{tabular}

3 Un mensaje de prioridad $i$ generado por el nodo $\boldsymbol{x}$ en el tiempo to debe ser recibido por el proceso situado en el nodo $y$ en el tiempo $t_{l}$, siendo $t_{0}-t_{l}=D i$ el tiempo de vida del mensaje. Sí $t_{0}-t_{l}>D i$ el mensaje deja de tener validez en el ámbito de las redes de control.

\section{RESPUESTA TEMPORAL PLANEADA: CRITERIO DE TINDELL}

En [2] los autores presentaron una solución formal para determinar el máximo tiempo de respuesta de una red CAN (análisis del peor caso), partiendo de un modelo en el cual:

- Los identificadores son establecidas según la vida útil del mensaje de modo tal que el de menor vida útil (el más urgente) tenga máxima prioridad.

- Para realizar el planeamiento, se asume que los mensajes arriban a los controladores CAN cíclicamente.

- Un mensaje dado aparece en una ventana de tiempo limitada por la máxima frecuencia de arribo.

El análisis para llegar a la expresión resultante dada en [2] es sencillo, si se consideran las peores circunstancias que contribuyen a la ocurrencia del máximo tiempo de bloqueo de un mensaje con prioridad $m$ en un sistema CAN de $g$ mensajes distintos $(m<g)$. Considérese el caso de $m=l$ (mensaje de mayor prioridad en el sistema). El máximo tiempo de acceso al bus para este mensaje se producirá cuando su arribo a la cola del procesador esté atrasado un tiempo de bit respecto al comienzo de la transmisión de un mensaje de menor prioridad y con máxima cantidad de bytes de datos. Expresado en forma compacta, el máximo tiempo de bloqueo que experimentará un mensaje de máxima prioridad en un sistema con $g$ mensajes distintos estará dado por:

$$
B_{1}=\operatorname{Max}\left[\sum_{i=2}^{g} C_{i}\right]
$$

donde $C_{i}$ representa el tiempo de transmisión del mensaje cuya prioridad es $i$.

Un mensaje con prioridad $m=2$ tendrá, en cambio, dos componentes de bloqueo. La primera, similar al caso anterior, producida por la presencia de un mensaje de menor prioridad $(2<m \leq g)$ y máximo tamaño en bytes de datos $\left(B_{2}\right)$; la segunda, la producirán la transmisión 
de paquetes de mayor prioridad $(m=1)$. Además, deben considerarse los nuevos arribos de la clase que afecta directamente su respuesta temporal (en este caso la clase $m=1$ ). Una manera de determinar el factor de bloqueo es mediante la siguiente expresión:

$$
t_{2}^{1} \approx B_{2}+\sum_{j=1}^{1}\left\lceil\frac{t_{2}^{0}}{T_{j}}\right\rceil C_{j}
$$

donde:

$t_{2}^{1} \quad$ tiempo de bloqueo del mensaje con prioridad 2 en la primera iteración.

$T_{j}$ tiempo entre arribos del mensaje $j$.

En el segundo miembro de la ecuación (2), $B_{2}$ representa el factor de bloqueo debido a un paquete de menor prioridad con máximo tamaño en bytes de datos, mientras que el segundo sumando toma en consideración el factor de bloqueo correspondiente a paquetes de mayor prioridad a la clase considerada. La función "cielo" dentro de la sumatoria retorna un entero que representa el número de veces que arriba el mensaje $j$ durante el tiempo de bloqueo del mensaje cuya prioridad es 2 .

Evidentemente, la ecuación resultante es iterativa. El subíndice respecto de $t$ en la ecuación (2) indica la prioridad del mensaje considerado, mientras que el superíndice denota el número de iteración efectuada.

Extendiendo el razonamiento para un paquete de prioridad $k(k \leq g)$, el tiempo de bloqueo estará dado por:

$$
t_{k}^{n} \approx B_{k}+\sum_{j=1}^{k-1}\left\lceil\frac{t_{k}^{n-1}}{T_{j}}\right\rceil C_{j}
$$

La expresión (3) es una representación aproximada del tiempo de bloqueo de un mensaje con prioridad $k$. Cuando se consideran otros aspectos relevantes del protocolo que, dependiendo del grado de precisión requerido, deben ser tenidos en cuenta, esta expresión se transforma en:

$$
t_{k}^{n} \approx B_{k}+\sum_{j \in h p(k)}\left\lceil\frac{t_{k}^{n}+J_{j}+\tau_{b i t}}{T_{j}}\right\rceil C_{j}
$$

donde:

$J_{j} \quad$ es el jitter en la cola (tiempo que demora en atender el procesador a un mensaje que arriba a la cola (buffer de recepción)).

$\tau_{\text {bit }} \quad$ Tiempo de 1 bit.

$h p(k)$ es el conjunto de mensajes con mayor prioridad que el mensaje $k$.

Tal como fue adelantado en la primera sección, el siguiente paso es obtener los máximos tiempos de respuesta para los mensajes de tiempo real en el sistema descrito por la tabla 1 y verificar el planeamiento de este tipo de mensajes. En el contexto de las redes CAN se define el tiempo de respuesta, $R i$, de un mensaje cuya prioridad es $i$ como:

$$
R_{i}=t_{i}+C_{i}
$$

donde $t_{i}$ es el tiempo de bloqueo del mensaje cuya prioridad es i, calculado según (3). Aplicando la ecuación (3) al sistema descrito en la Tabla 1 se obtiene el resultado mostrado en la Tabla 2. Observando la columna Ri, se puede constatar que el tiempo de respuesta de los mensajes es menor que la vida útil de los mismos, Di; por lo tanto, el sistema bajo estudio es susceptible de ser planificado:

Tabla 2 Tiempos de respuesta (criterio de Tindell).

\begin{tabular}{|c|c|c|l|l|}
\hline $\operatorname{Pr}$ & Bytes & Di (s) & Ci(s) & $\mathrm{Ri}_{125 \mathrm{Kbps}}$ \\
\hline 1 & 4 & 0.005 & $7,6 \mathrm{E}-4$ & 0,0014 \\
\hline 2 & 1 & 0.005 & $5,2 \mathrm{E}-4$ & 0,00192 \\
\hline 3 & 1 & 0.005 & $5,2 \mathrm{E}-4$ & 0,00244 \\
\hline 4 & 2 & 0.005 & $6 \mathrm{E}-4$ & 0,00304 \\
\hline 5 & 1 & 0.005 & $5,2 \mathrm{E}-4$ & 0,00356 \\
\hline 6 & 1 & 0.005 & $5,2 \mathrm{E}-4$ & 0,00408 \\
\hline 7 & 1 & 0.005 & $5,2 \mathrm{E}-4$ & 0,0046 \\
\hline
\end{tabular}

Sin embargo, el cálculo, según [2] para el sistema descrito por la tabla 1 (considerando todos los mensajes), no es planificable y exigiría un aumento de la tasa de transmisión.

\section{RELAJACIÓN DE LA RESTRICCIÓN DEL CRITERIO TINDELL: ANÁLISIS DE VALOR MEDIO}

En este punto resta encontrar los tiempos promedios de respuesta para aquellos mensajes que no tienen restricciones de tiempo real (prioridad 7 y 8 en tabla 1). Considerando que el proceso de arribo de este tipo de mensajes responde a un proceso de Poisson de parámetros $\lambda_{i} t$, con $\lambda_{i}=1 / T_{i}$, es posible utilizar los resultados dados en [4] y resumidos a continuación: 


$$
\begin{gathered}
\bar{R}_{1}=\frac{1}{2} \sum_{i=2}^{G} \lambda_{i} C_{i}^{2}+C_{1} \\
\bar{R}_{i}=\frac{\bar{R}_{i-1}\left(1-\sigma_{i-2}+\rho_{i-1}\right)}{\left(1-\sigma_{i-1}\right)}+C_{i}
\end{gathered}
$$

donde:

$$
\begin{array}{ll}
\rho_{i}\left(=\lambda_{i} C_{i}\right) & \begin{array}{l}
\text { Factor de utilización del canal por usuarios } \\
\text { con prioridad } i \text {. }
\end{array} \\
\bar{R}_{i} & \begin{array}{l}
\text { es el tiempo promedio de bloqueo de un } \\
\text { mensaje cuya prioridad es } i .
\end{array} \\
\sigma_{i}\left(=\sum_{j=1}^{i} \rho_{j}\right) & \begin{array}{l}
\text { factor de utilización del canal por usuarios } \\
\text { con prioridades } 1 \text { a } i .
\end{array}
\end{array}
$$

Aplicando (5) y (6) al sistema descrito en la Tabla 1, se obtienen los resultados mostrados en la Tabla 3.

Tabla 3 Valor promedio de los tiempos de respuesta.

\begin{tabular}{|c|c|c|c|c|l|}
\hline $\operatorname{Pr}$ & Bytes & $\lambda_{\mathrm{i}}$ & $\mathrm{Di}(\mathrm{s})$ & $\mathrm{Ci}(\mathrm{s})$ & $\mathrm{Ri}_{125 \mathrm{Kbps}}$ \\
\hline 1 & 4 & 20 & 0.005 & $7,6 \mathrm{E}-4$ & 0,001784 \\
\hline 2 & 1 & 200 & 0.005 & $5,2 \mathrm{E}-4$ & 0,00156 \\
\hline 3 & 1 & 200 & 0.005 & $5,2 \mathrm{E}-4$ & 0,001669 \\
\hline 4 & 2 & 200 & 0.005 & $6 \mathrm{E}-4$ & 0,001884 \\
\hline 5 & 1 & 200 & 0.005 & $5,2 \mathrm{E}-4$ & 0,002003 \\
\hline 6 & 1 & 200 & 0.005 & $5,2 \mathrm{E}-4$ & 0,002238 \\
\hline 7 & 1 & 200 & 0.005 & $5,2 \mathrm{E}-4$ & 0,002561 \\
\hline 8 & 3 & 200 & $0.005 *$ & $6,8 \mathrm{E}-4$ & 0,00319 \\
\hline 9 & 3 & 200 & $0.005 *$ & $6,8 \mathrm{E}-4$ & 0,004186 \\
\hline
\end{tabular}

En la Tabla 3, los campos sombreados muestran la información relevante para esta propuesta. En ellos se puede observar que $\mathrm{Ri}<\mathrm{Di}$ (en promedio). La información que brinda la desigualdad $\mathrm{Ri}<\mathrm{Di}$ no es relevante a la hora de evaluar la cantidad de mensajes perdidos y para ello es necesario el cálculo de la varianza de los procesos de arribo para los mensajes de interés (en este caso mensajes de prioridad 8 y 9 ).

\section{CONCLUSIONES}

En la actualidad, cuando se diseña un sistema de control distribuido, se tiene como premisa el planeamiento de todas las tareas, sin importar el tipo de mensaje de que se trate [2]. La consecuencia de esta política es que se debe aumentar la tasa de transmisión. El aumento de la tasa de transmisión trae consigo dificultades de sincronización y lectura correcta de los datos por los nodos receptores. Los errores causan retransmisiones, cuyo efecto es el de hacer más aguda la necesidad de aumentar la tasa de transmisión, para un correcto planeamiento [3].

El objetivo de este trabajo fue proveer una estrategia mixta: criterios de planeamiento a mensajes con características de tiempo real y análisis de valor medio a aquellos mensajes que admiten retardos y hasta algún nivel de pérdidas, sin que por ello afecte el normal funcionamiento del sistema. A base de medidas estadísticas, tal como el promedio y la varianza de los procesos de arribo en el bus, es posible predecir el número de mensajes perdidos. El modelado de la aparición de mensajes en una red CAN de acuerdo a una distribución de Poisson no es nuevo [6]. Sin embargo, este modelo, aplicado estrictamente, tiene la limitación de que no asegura que los mensajes de baja prioridad puedan transmitirse sin haber perdido su vigencia dado que el acceso al bus es priorizado. El análisis de valor medio [4] brinda información relevante a la hora del diseño, pues permite evaluar a priori el comportamiento del sistema y ajustar la tasa de transmisión, si así fuera necesario. En consecuencia, se postula en este trabajo que se ha desarrollado un modelo que permite negociar la calidad de servicio.

\section{AGRADECIMIENTOS}

Esta publicación fue posible gracias al proyecto UTFSM 230322, y gracias al proyecto de la Fundación Andes $\mathrm{N}^{\mathrm{o}} \mathrm{C}-13755 / 9$.

\section{REFERENCIAS}

[1] K. Tindell and A. Burns. "Guaranteeing Message Latencies on Control Area Network (CAN)". Proc. of the 1st. International CAN Conference (ICC'94).

[2] K. Tindell, A. Burns and A. Wellings. "Calculating Controller Area Network (CAN) Message Response Times". Control Engineering Practice. Vol. 3. No 8, pp. 1163-1169. 1995.

[3] T. Nolte. "Reducing Pessimism and Increasing Flexibility in the Controller Area Network", Mälardalen University Licentiate Thesis. 2003.

[4] A. Crespo. "Redes de Control: Análisis y Simulación del Protocolo CAN en un Escenario Real". Tesis para optar al grado de Master. Universidad Técnica Federico Santa María. Valparaíso. Chile. 2000. 
[5] R. Bosch GmbH. "CAN Specification Version 2.0". Postfach 50. D7000, Stuttgart, Germany. 1991.

[6] Z. Wang, H. Lu, G.E. Hedrick and M. Stone. "Message Delay Analysis for CAN Based
Networks". Proceedings of the 1992 ACM/ SIGAPP Symposium on Applied computing: technological challenges of the 1990's, pp. 89-94. 1992. 